\section{APOE- 44 allele predisposes carotid endarterectomy patients to cognitive dysfunction}

Approximately $25 \%$ of patients undergoing carotid endarterectomy (CEA) experience anesthesia-unrelated cognitive dysfunction immediately afterwards; many show continued dysfunction a month later. The presence of the apolipoprotein $\mathrm{E}(A P O E)-\varepsilon 4$ allele has been associated with worse outcome after traumatic and ischemic brain injuries. To determine whether this allele can be used to improve selection of patients for CEA, Heyer and colleagues studied the relationship between presence of the allele and development of neurocognitive impairment in 75 prospectively recruited patients undergoing CEA. Patients undergoing lumbar laminectomy $(n=46)$, matched for age and education, acted as the control group with regard to surgical anesthesia.

$A P O E$ genotype was determined using polymerase chain reaction amplification of restriction fragment length polymorphisms. Patients undertook five neuropsychological tests before and 1 month after surgery to produce a total deficit score.

Frequency of the $A P O E-\varepsilon 4$ allele was similar to that in the general population, with $12 / 75$ CEA patients (16\%) carrying it; 5 of these experienced cognitive deficit 30 days after surgery, compared with $3 / 63$ without the allele $(P=0.002)$. Furthermore, carriers' deficits were greater than those of patients in whom the APOE- $\varepsilon 4$ allele was absent $(P=0.02)$. Multivariate analysis showed that having the $A P O E-\varepsilon 4$ allele resulted in a greatly increased risk of neurocognitive dysfunction 1 month after CEA (odds ratio 62.28); obesity and diabetes mellitus were also independent predictors (odds ratios 24.43 and 51.42, respectively).

The allele's strong, independent association with cognitive impairment means that it could be used to help identify patients for whom risk of cognitive impairment outweighs the benefit of CEA.

Kate Matthews

Original article Heyer EJ et al. (2005) APOE- $\varepsilon 4$ predisposes to cognitive dysfunction following uncomplicated carotid endarterectomy. Neurology [doi: 10.1212/

01.wnl.0000184579.23624.6b]

\section{The risk of developing parkinsonism}

A recent retrospective cohort study using data from Ontario, Canada found that, contrary to previous suggestions, atypical antipsychotic drugs are not always associated with a lower risk of parkinsonism than typical antipsychotics.

Rochon and colleagues identified 57,838 Ontario residents aged 66 years or older who had dementia with no evidence of schizophrenia or major depressive disorder. Of these, 25,769 were being treated with antipsychotic therapy-11,571 (45\%) were receiving an atypical antipsychotic (e.g. risperidone) and 14,198 (55\%) a typical antipsychotic (e.g. haloperidol). The subjects were assessed for evidence of parkinsonism during a period of continuous antipsychotic use lasting up to 1 year; those not receiving antipsychotic treatment acted as controls. The researchers used Cox proportional hazard models to study whether there was an association between the occurrence of parkinsonism and the type, dose and potency of the drug being taken by a patient.

Although patients receiving a typical antipsychotic were $30 \%$ more likely overall to develop parkinsonism than those receiving an atypical antipsychotic (adjusted hazard ratio $1.30 ; 95 \% \mathrm{Cl} 1.04-1.58)$, when the researchers looked at those treated with a high-dose atypical antipsychotic, they found that there was a similar risk of parkinsonism development for these patients and those receiving a typical antipsychotic $(P=0.7)$.

The authors conclude that the dispensing of atypical antipsychotics, particularly when given at a high dose, should be undertaken with caution because of the risk of developing parkinsonism.

Christine Kyme

Original article Rochon PA et al. (2005) Atypical antipsychotics and parkinsonism. Arch Intern Med 165: 1882-1888

\section{Candidate gene for Tourette's syndrome}

A recent genetic study investigating individuals with Tourette's syndrome (TS) has uncovered rare mutations in a gene called Slit and Trk-like 1 (SLITRK1) that are present in a small subset of patients affected with the condition. 